\title{
LINEAR DIFFERENTIAL OPERATOR WITH AN INVOLUTION AS A GENERATOR OF AN OPERATOR GROUP
}

\author{
Anatoly G. Baskakov, Ilya A. Krishtal and Natalia B. Uskova
}

Abstract. We use the method of similar operators to study a mixed problem for a differential equation with an involution and an operator-valued potential function. The differential operator defined by the equation is transformed into a similar operator that is an orthogonal direct sum of simpler operators. The result is used to construct an operator group that describes the mild solutions of the original problem. It may also serve as a justification for the use of the Fourier method to solve it.

Mathematics subject classification (2010): 35L75, 35Q53, 37K10, 37K35.

Keywords and phrases: Spectral asymptotic analysis, method of similar operators, mixed problem, operator groups.

\section{REFERENCES}

[1] W. Arendt, C. J. K. Batty, M. Hieber, AND F. NeubRAnder, Vector-valued Laplace transforms and Cauchy problems, vol. 96 of Monographs in Mathematics, Birkhäuser/Springer Basel AG, Basel, second ed., 2011.

[2] A. BASKAKOV AND V. DidenKo, Spectral analysis of differential operators with unbounded periodic coefficients, Differential Equations 51 (2015), pp. 325-341.

[3] A. G. BASKAKOV, Spectral tests for the almost periodicity of the solutions of functional equations, Mat. Zametki 24 (1978), pp. 195-206, 301, English translation: Math. Notes 24 (1978), no. 1-2, pp. 606-612 (1979).

[4] A. G. BASKAKOV, Methods of abstract harmonic analysis in the theory of perturbations of linear operators, Sibirsk. Mat. Zh. 24 (1983), pp. 21-39, 191, English translation: Siberian Math. J. 24 (1983), no. 1, pp. 17-32.

[5] A. G. BASKAKOV, The Krylov-Bogolyubov substitution in the theory of perturbations of linear operators, Ukrain. Mat. Zh. 36 (1984), pp. 606-611, English translation: Ukrainian Math. J. 36 (1984), no. $5,451-455$.

[6] A. G. BASKAKOV, A theorem on splitting of an operator and some related problems in the analytic theory of perturbations, Izv. Akad. Nauk SSSR Ser. Mat. 50 (1986), pp. 435-457, 638, English translation: Math. USSR-Izv. 28 (1987), no. 3, pp. 421-444.

[7] A. G. BASKAKov, Spectral analysis of perturbed non-quasi-analytic and spectral operators, Izv. Ross. Akad. Nauk Ser. Mat. 58 (1994), pp. 3-32, English translation: Russian Acad. Sci. Izv. Math. 45 (1995), no. 1, pp. 1-31.

[8] A. G. BASKAKOV, Estimates for the Green's function and parameters of exponential dichotomy of a hyperbolic operator semigroup and linear relations, Mat. Sb. 206 (2015), pp. 23-62, English translation: Sb. Math. 206 (2015), no. 8, pp. 1049-1086.

[9] A. G. Baskakov, A. V. Derbushev, And A. O. Shcherbakov, The method of similar operators in the spectral analysis of the nonselfadjoint Dirac operator with nonsmooth potential, Izv. Ross. Akad. Nauk Ser. Mat. 75 (2011), pp. 3-28, English translation: Izv. Math. 75 (2011), no. 3, 445-469.

[10] A. G. Baskakov, L. Y. Kabantsova, I. D. Kostrub, And T. I. Smagina, Linear differential operators and operator matrices of the second order, Differ. Equ. 53 (2017), pp. 8-17, Translation of Differ. Uravn. 53 (2017), no. 1, 10-19. 
[11] A. G. BASKaKov AND I. A. KRishtal, On completeness of spectral subspaces of linear relations and ordered pairs of linear operators, J. Math. Anal. Appl. 407 (2013), pp. 157-178.

[12] A. G. BASKAKOV AND I. A. KRISHTAL, Spectral analysis of abstract parabolic operators in homogeneous function spaces, ii, Mediterranean Journal of Mathematics 14 (2017), p. 181.

[13] A. G. Baskakov, I. A. Krishtal, And E. Y. Romanova, Spectral analysis of a differential operator with an involution, Journal of Evolution Equations 17 (2017), pp. 669-684.

[14] A. G. BASKAKOV AND D. M. Polyakov, The method of similar operators in spectral analysis for the Hill operator with nonsmooth potential, Mat. Sb. 208 (2017), pp. 3-47, English Translation: Sb. Math., 2017, 208 (1): 1-43.

[15] M. S. BuRLutS KAYA AND A. P. Khromov, The Fourier method in a mixed problem for a first-order partial differential equation with involution, Zh. Vychisl. Mat. Mat. Fiz. 51 (2011), pp. 2233-2246, English translation: Comput. Math. Math. Phys. 51 (2011), no. 12, 2102-2114.

[16] M. S. BurlutskayA AND A. P. Khromov, Mixed problems for first-order hyperbolic equations with involution, Dokl. Akad. Nauk 441 (2011), pp. 156-159, English translation: Dokl. Math. 84 (2011), no. 3, 783-786.

[17] M. S. Burlutskaya AND A. P. Khromov, Functional-differential operators with involution and Dirac operators with periodic boundary conditions, Dokl. Akad. Nauk 454 (2014), pp. 15-17, English translation: Dokl. Math. 89 (2014), no. 1, 8-10.

[18] M. S. Burlutskaya, V. P. Kurdyumov, And A. P. Khromov, Refined asymptotic formulas for the eigenvalues and eigenfunctions of the Dirac system, Dokl. Akad. Nauk 443 (2012), pp. 414-417, English translation: Dokl. Math. 85 (2012), no. 2, 240-242.

[19] C. Chicone AND Y. LATUSHKin, Evolution semigroups in dynamical systems and differential equations, vol. 70 of Mathematical Surveys and Monographs, American Mathematical Society, Providence, RI, 1999.

[20] N. Dunford AND J. T. SChwartz, Linear Operators. I. General Theory, with the assistance of W. G. Bade and R. G. Bartle, pure and Applied Mathematics, vol. 7, Interscience Publishers, Inc., New York, 1958.

[21] K.-J. ENGEL AND R. NAGEL, One-parameter semigroups for linear evolution equations, vol. 194 of Graduate Texts in Mathematics, Springer-Verlag, New York, 2000, with contributions by S. Brendle, M. Campiti, T. Hahn, G. Metafune, G. Nickel, D. Pallara, C. Perazzoli, A. Rhandi, S. Romanelli and R. Schnaubelt.

[22] K. O. FRIEDRICHS, Lectures on advanced ordinary differential equations, notes by P. Berg, W. Hirsch, P. Treuenfels, Gordon and Breach Science Publishers, New York, 1965.

[23] I. Gohberg, M. A. KAAshoEK, AND J. Kos, Classification of linear time-varying difference equations under kinematic similarity, Integral Equations Operator Theory 25 (1996), pp. 445-480.

[24] E. Hille AND R. S. Phillips, Functional analysis and semi-groups, American Mathematical Society Colloquium Publications, vol. 31, American Mathematical Society, Providence, R. I., 1957, rev. ed.

[25] R. E. Kalman AND R. S. BuCy, New results in linear filtering and prediction theory, Trans. ASME Ser. D. J. Basic Engrg. 83 (1961), pp. 95-108.

[26] A. P. Khromov, Equiconvergence theorems for integro-differential and integral operators, Mat. Sb. (N. S.) 114 (156) (1981), pp. 378-405, 479.

[27] Y. LATUSHKIN AND S. MontgomerY-SMith, Evolutionary semigroups and Lyapunov theorems in Banach spaces, J. Funct. Anal. 127 (1995), pp. 173-197.

[28] A. M. LJapunov, Sobranie sochineniǔ. Tom II, Izdat. Akad. Nauk SSSR, Moscow, 1956.

[29] B. Mityagin, Spectral expansions of one-dimensional periodic Dirac operators, Dyn. Partial Differ. Equ. 1 (2004), pp. 125-191.

[30] M. NieZABitows Ki, Kinematic similarity of the discrete linear time-varying systems, in 2015 20th International Conference on Control Systems and Computer Science, May 2015, pp. 10-17.

[31] V. A. PLISs, Nelokalnye problemy teorii kolebanii, Izdat. "Nauka", Moscow, 1964.

[32] V. A. PLISs, Families of periodic solutions of systems of differential equations of second order without dissipation, Differencial'nye Uravnenija 1 (1965), pp. 1428-1448.

[33] D. M. Polyakov, Spectral properties of an even-order differential operator, Differ. Equ. 52 (2016), pp. 1098-1103, translation of Differ. Uravn. 52 (2016), no. 8, 1133-1137. 
[34] M. A. Sadybekov And A. M. Sarsenbi, Criterion for the basis property of the eigenfunction system of a multiple differentiation operator with an involution, Differ. Equ. 48 (2012), pp. 1112 1118, translation of Differ. Uravn. 48 (2012), no. 8, 1126-1132.

[35] Y. TomiLov, A resolvent approach to stability of operator semigroups, J. Operator Theory 46 (2001), pp. 63-98.

[36] R. E. L. Turner, Perturbation of compact spectral operators, Comm. Pure Appl. Math. 18 (1965), pp. 519-541.

[37] N. B. Uskova, On a result of R. Turner, Mat. Zametki 76 (2004), pp. 905-917, English translation: Math. Notes 76 (2004), no. 5-6, 844-854.

[38] N. B. Uskova, On spectral properties of Sturm-Liouville operator with matrix potential, Ufa Math. J. 7 (2015), pp. 84-94.

[39] N. B. UsKova, On the spectral properties of a second-order differential operator with a matrix potential, Differ. Equ. 52 (2016), pp. 557-567, translation of Differ. Uravn. 52 (2016), no. 5, 579-588.

[40] V. S. Vladimirov, Equations of mathematical physics, "Mir", Moscow, 1984, translated from the Russian by Eugene Yankovsky [E. Yankovskiī]. 